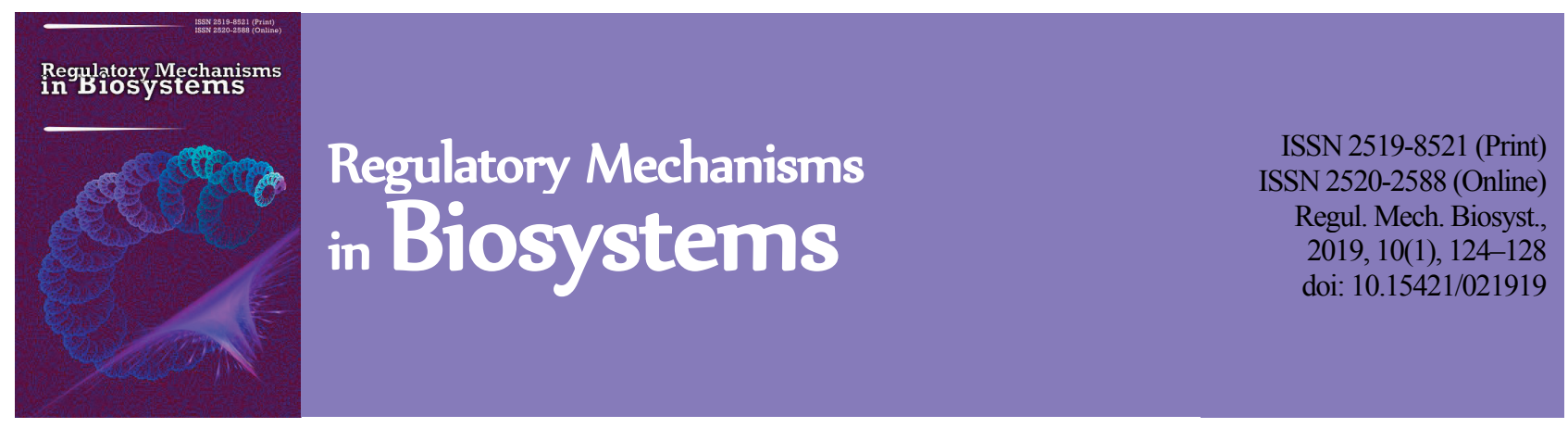

\title{
Bifidogenic properties of cell-free extracts derived from probiotic strains of Bifidobacterium bifidum and Lactobacillus reuteri
}

\author{
O. V. Knysh \\ SI "I. I. Mechnikov Institute of Microbiology and Immunology of National Academy of Medical Sciences of Ukraine", Kharkiv, Ukraine
}

Article info

Received 04.01.2019

Received in revised form 07.02.2019

Accepted 10.02.2019

SI "I. I. Mechnikov Institute of Microbiology and Immunology of National Academy of Medical Sciences of Ukraine”, Pushkins ka st., 14/16, Kharkiv, 61057, Ukraine. Tel.: +38-068-210-47-10.

E-mail:knysh_oksana@ukr.net

\begin{abstract}
Knysh, O. V. (2019). Bifidogenic properties of cell-free extracts derived from probiotic strains of Bifidobacterium bifidum and Lactobacillus reuteri. Regulatory Mechanisms in Biosystems, 10(1), 124-128. doi:10.15421/021919
\end{abstract}

Comprehensive study of the biological activity of structural components and metabolites of "beneficial" microorganisms opens the prospects of efficient and rational use of their biotechnological potential in the correction of microecological and related disorders. The study tested proliferative activity and biofilm formation by Bifidobacterium bifidum probiotic strain under the influence of cell-free extracts containing structural components and metabolites of the probiotic strains of B. bifidum and Lactobacillus reuteri. Cell-free extracts were obtained by disintegrating suspensions of probiotic cells by cyclic freezing-thawing, cultivating probiotic microorganisms in their own disintegrates and subsequent filtration of the obtained disintegrates and cultures. The proliferative activity and biofilm formation of the probiotic test culture were studied by spectrophotometric microtiter plate method with $10 \% \mathrm{vol}, 30 \% \mathrm{vol}$ and $50 \% \mathrm{vol}$ content of cell-free extracts in the cultivation medium. All investigated extracts showed a significant concentration-dependent stimulatory effect on the proliferative activity of $B$. bifidum. According to the degree of stimulatory effect on the B. bifidum proliferation, cell-free extracts arranged in ascending order: MLG (filtrate of $L$. reuteri culture, grown in $L$. reuteri disintegrate supplemented with $0.8 \mathrm{M}$ glycerol and $0.4 \mathrm{M}$ glucose) $<\mathrm{MB}$ (filtrate of $B$. bifidum culture, grown in B. bifidum disintegrate) $<\mathrm{B}$ (filtrate of $B$. bifidum disintegrate) $<\mathrm{ML}$ (filtrate of $L$. reuteri culture, grown in $L$. reuteri disintegrate) $<\mathrm{L}$ (filtrate of $L$. reuteri disintegrate). With the same content in the culture medium, filtrates of disintegrates had a more pronounced stimulatory effect than filtrates of cultures grown in their own disintegrates. Cell-free extracts from $L$. reuteri (L and ML) exerted a more pronounced stimulatory effect than cell-free extracts from B. bifidum. Not all studied cell-free extracts stimulated the biofilm formation by B. bifidum. The effect of cell-free extracts on this process depended on their type and concentration. Extract L had a predominantly inhibitory effect on biofilm formation by B. bifidum. The most pronounced stimulatory effect on biofilm formation by B. bifidum came from extract MLG. ML, B and MB extracts stimulated this process approximately equally. The detection of significant bifidogenic effect of the studied cell-free extracts may contribute to their pharmaceutical applications. Cell-free extracts can be used as metabiotics or prebiotics for increasing the survival of the injected probiotic, facilitating its inoculation in the gastrointestinal tract when used together. The obtained data encourage further careful study of the biochemical composition of cell-free extracts and efforts to clarify the mechanism of their action.

Keywords: probiotic bacteria; biofilm formation; biotechnological potential; proliferative activity; cell-free extract.

\section{Introduction}

The gut microbiota is involved in a wide variety of physiological processes: degradation of non-digestible food compounds, absorption of nutrients, metabolism of bile acids and xenobiotics, production of essential vitamins, bioactive metabolites and signaling molecules. It has a strong influence on the intestinal epithelium and immune system development, host behaviour and metabolism. Recent studies have revealed a correlation between the composition of the microbiota and diseases such as obesity, metabolic syndrome, diabetes, cardiovascular and cerebrovascular diseases: hypertension and atherosclerosis, liver disease, malnutrition, inflammatory bowel disease, rheumatoid arthritis, cancer, autism, and Parkinson's disease (Landman \& Quevrain, 2016; Wang et al., 2017; Feng et al., 2018).

The gut microbiota of a healthy person is characterized by the dominance of obligate anaerobic members of the phyla Firmicutes, Bacteroidetes and Actinobacteria (Kho \& Lal, 2018). An increase in the number of facultative anaerobic Enterobacteriaceae (phylum Proteobacteria) is a marker of gut dysbiosis (Litvak et al., 2017). Members of the genus Bifidobacterium play a central role in maintaining and restoring gut homeostasis (O'Callaghan \& van Sinderen, 2016; Rivière et al., 2016; Sar- kar \& Mandal, 2016). Bifidobacterium bifidum, B. longum and B. breve are the predominant microbes that colonize the intestine in the first days after birth. They constitute $40-80 \%$ of the gut microbiota of newborns. In infants from 6 months of age, the total diversity of bacteria increases, but the proportion of bifidobacteria decreases to $30-40 \%$. In childhood and adolescence, the number of bifidobacteria continues gradually to decline. In adults, their content varies from 0 to $25 \%$. The elderly phase of life is characterized by a further decrease in the number of bifidobacteria. High content of bifidobacteria in the gut microbiota is associated with a 'healthy' state and longevity. The level of bifidobacteria is a biomarker of gut flora health and can be influenced by nutrition, use of antibiotics and other drugs (Valdes et al., 2018). The loss of bifidobacteria is harmful for the health of the host: it adversely affects the remaining microorganisms, increases the risk of invasion and colonization of the gut epithelium by pathogenic and opportunistic microorganisms (Sarkar \& Mandal, 2016; Rivière et al., 2016; O’Neillet al., 2017).

B. bifidum along with other bifidobacteria provides mucosal colonization resistance. Antagonistic activity against many opportunistic and obligatory pathogenic microorganisms is realized through the synthesis of antimicrobial substances: bateriocins, exopolysaccharides, organic acids. B. bifidum can secrete molecules that inhibit the expression of virulence 
genes responsible for colonization and systemic dissemination of intestinal pathogens. These beneficial microorganisms not only enhance the gut barrier, but also reduce the risk of occurrence of upper respiratory infections. B. bifidum strains play a key role in the evolution, maturation of the immune system and modulation of the host immune response. Bifidobacteriummediated immune modulation is considered as one of the new methods of treatment (Ku et al., 2016; Quigley, 2017; O’Neill et al., 2017).

Recently there has been a growing interest in compounds that positively influence the composition and activity of gut microbiota. The most common way to obtain a bifidogenic effect is to consume probiotics and prebiotics (Rivière et al., 2016). Bifidobacterial growthpromoting factors of several groups are known: 1) breast and cow milk components ( $\kappa$-casein, caseinomacropeptide, enzymatically digested $\kappa$ casein), 2) oligosaccharides (for example, raffinose, stachyose, lactulose), 3) polysaccharides (for example, inulin), 4) alcohol saccharides (for example, lactitol), 5) quinones (for example, 2-amino-3-carboxy-1,4naphthoquinone) (Cicvarek et al., 2011). High expectations are pinned on microbial products as a means to renovate balance of intestinal microbial populations (Hamet et al., 2016; Richards et al., 2016; Gagliardi et al., 2018). Use of the biological activity of the probiotics' derivatives is considered as an alternative approach to prevention of infections by enriching colonization with protective commensal species and enhancing the protective properties of the immune system. This approach can lead to interesting therapeutic results (Landman \& Quevrain, 2016; Turroni et al., 2017; Keith \& Pamer, 2018). Therapy with preparations based on probiotics' structural components and metabolites can replace insufficiently effective intact cell probiotics therapy in the future (Shenderov, 2013; Sarkar \& Mandal, 2016; Singh et al., 2018). In addition, the determination and use of substances that have a stimulatory effect on probiotic cultures has a commercial advantage, since it will increase the overall productivity of cellular biomass. This circumstance is of particular importance in conditions of lower productivity and higher cost of Bifidobacterium spp. production as compared with other aerobic or facultative anaerobic microorganisms (Ku et al., 2016).

Considering that the beneficial effect of probiotic bacteria is due to the biological activity of their structural components and metabolites, our efforts were aimed at obtaining them. We have developed a method for the obtaining of cell-free extracts, containing biologically active probiotics' derivatives (Knysh et al., 2018). The applied method of slow disintegration provides access to the extracellular space of the structural components and metabolites of the probiotic cells, which are subjected to stress and damaging factors during the freeze-thaw stages. The resulting disintegrate, in addition to viable cells that survived repeated freezethaw cycles, contains substances suitable for bacterial nutrition. This allows the use of the disintegrate as a nutrient medium for the cultivation to receive metabolites of actively proliferating probiotic bacteria.

The aim of the research was to investigate the influence of cell-free extracts, contained derivatives of $L$. reuteri and $B$. bifidum on proliferative activity and biofilm formation by $B$. bifidum in vitro.

\section{Materials and methods}

The effect of five cell-free extracts containing probiotic derivatives on the proliferation and biofilm formation by B. bifidum was investigated. These were:

- L - filtrate of L. reuteri disintegrate;

- ML - filtrate of $L$. reuteri culture, grown in L. reuteri disintegrate;

- MLG - filtrate of L. reuteri culture, grown in L. reuteri disintegrate supplemented with $0.8 \mathrm{M}(73.7 \mathrm{~g} / \mathrm{l})$ glycerol and $0.4 \mathrm{M}(72.1 \mathrm{~g} / \mathrm{l})$ glucose;

- B - filtrate of B. bifidum disintegrate;

- MB - filtrate of B. bifidum culture, grown in B. bifidum disintegrate.

Disintegrates and cultures of probiotics were obtained using the method developed by the authors (Knysh et al., 2018). Cell-free extracts contained structural components and metabolites of the commercial strains B. bifidum 1 (from medical product "Bifidumbacterin-Biopharma", JSC Biopharma, Ukraine) and L. reuteri DSM 17938 (from medical product "BioGaia", BioGaia Production AB, Sweden). The commercial strain
B. bifidum 1 was used as a proliferating and biofilm-forming test culture. The lyophilized microbial mass was rehydrated and recovered by cultivation in tryptone soya broth (TSB; HiMedia, India) anaerobically for 24 hours at $37^{\circ} \mathrm{C}$. After checking the purity of the culture, the cells were harvested by centrifugation for $10 \mathrm{~min}$ at $3000 \mathrm{~g}$ and the pellet was washed twice with sterile isotonic saline solution to remove nutrient components. Finally, the pellet was resuspended in physiological saline solution $(0.9 \% \mathrm{NaCl})$ and an inoculum was prepared from it. The inoculum was a microbial suspension in a physiological saline solution with an optical density of 10 units on the McFarland scale $\left(\sim 10^{8} \mathrm{CFU} / \mathrm{ml}\right)$. The turbidity of the suspension was measured using the Densi-La-Meter II device (PLIVA-Lachema Diagnostika, Czech Republic).

The study of the effect of cell-free extracts on the B. bifidum proliferation was performed in sterile 96-well polystyrene microtiter plates (JSC "Eximcargotrade", Ukraine). TSB supplemented with 1\% glucose, cell-free extracts and inoculums were added into the test wells in the ratio of $8: 1: 1 ; 6: 3: 1$ and $4: 5: 1$. Thus, the final concentration of each extract in the cultivation medium was $10 \%$ vol, $30 \%$ vol or $50 \%$ vol. TSB supplemented with $1 \%$ glucose, physiological saline $(\mathrm{PhS})$ and inoculums were added into the positive control wells (PC) in the same ratio: $\mathrm{PC}_{10}\left(10 \%\right.$ vol of the $\mathrm{PhS}$ in the cultivation medium); $\mathrm{PC}_{30}$ (30\%vol of the $\mathrm{PhS}$ in the cultivation medium); $\mathrm{PC}_{50}(50 \% \mathrm{vol}$ of the $\mathrm{PhS}$ in the cultivation medium). The final concentration of microbial cells in the test and positive control wells was $\sim 10^{7} \mathrm{CFU} / \mathrm{ml}$. The negative control wells (NC) contained only TSB. The plates were covered with lids and incubated anaerobically for 24 hours at $35-37{ }^{\circ} \mathrm{C}$ in static conditions. Optical density (OD) of the wells was measured at $578 \mathrm{~nm}$ using a microtiter-plate reader "LisaScanEM" ("ErbaLachemas.r.o.", Czech Republic). The inhibition (or stimulation) indices were calculated by the formula: II (SI) $=\left(\Delta \mathrm{OD}-\Delta \mathrm{OD}_{\mathrm{PC}}\right) \div \Delta \mathrm{OD}_{\mathrm{PC}} \times 100 \%$, where $\Delta \mathrm{OD}$ and $\Delta \mathrm{OD}_{\mathrm{PC}}$ are changes in the optical density of the test and control samples within 24 hours, respectively.

The study of the effect of cell-free extracts on the biofilm formation by $B$. bifidum. After measuring the optical density of the wells, the plates continued to be incubated anaerobically in static conditions for 24 hours at $35-37^{\circ} \mathrm{C}$. Then the contents of wells were decanted. Each well was washed three times with sterile $0.1 \mathrm{M}$ phosphate-buffered saline (PBS; $\mathrm{pH} 7.2$ ). Attached biofilms were fixed by exposing them to hot air at $60{ }^{\circ} \mathrm{C}$ for $60 \mathrm{~min}$; they were stained by $1 \%$ crystal violet for 15 min. After that, the wells were washed ten times with distilled water. Ethanol as an eluent was gently added for resolubilization of the dye and after that microtiter plate covered with the lid. It was left at room temperature for $30 \mathrm{~min}$. Optical density (OD) of the eluate in the test and control was were measured at $630 \mathrm{~nm}$ by using a microtiter-plate reader "Erba Lisa Scantm EM" (Germany). Based on the OD values obtained for $\mathrm{NC}$ and test samples, biofilm formation was defined as:

- weak $\left(\mathrm{OD}_{\mathrm{NC}}<\mathrm{OD} \leq 2 \mathrm{xOD} \mathrm{NC}_{\mathrm{NC}}\right)$;

- moderate $\left(2 \mathrm{xOD}_{\mathrm{NC}}<\mathrm{OD} \leq 4 \mathrm{xOD} \mathrm{NC}_{\mathrm{NC}}\right)$;

- strong $\left(4 \mathrm{xOD}_{\mathrm{NC}}<\mathrm{OD}\right)$;

- no biofilm formation $\left(\mathrm{OD} \leq \mathrm{OD}_{\mathrm{NC}}\right)$, according to the description Lee et al. (2017).

The inhibition (or stimulation) indices were calculated by the formula: $\mathrm{II}(\mathrm{SI})=\left(\mathrm{OD}-\mathrm{OD}_{\mathrm{PC}}\right) \div \mathrm{OD}_{\mathrm{PC}} \times 100 \%$, where $\mathrm{OD}$ and $\mathrm{OD}_{\mathrm{PC}}$ are optical density of the test and control samples, respectively.

All experiments were performed three times. Each sample was tested in triplicate. The obtained data were statistically processed with Excel 2010 software (Microsoft, USA). Average values of obtained indices (x) with standard deviations (SD) were determined.

\section{Results}

First of all, it should be noted that an increase in the volume of the $\mathrm{PhS}$ replacing the nutrient medium in the control sample from $10 \%$ to $30 \%$ and from $10 \%$ to $50 \%$, is accompanied by a decrease in the optical density gain of the culture by an average of $17 \%$ and $73.6 \%$, respectively (Fig. 1). This is a dilution effect due to a decrease in the amount of nutrients in the cultivation medium. Addition to the cultivation medium of $\mathrm{L}$ and MLcell-free extracts at a concentration of $10 \%$ leads to a significant increase in the optical density gain of the culture compared to $\mathrm{PC}_{10}(\mathrm{SI}$ 
are $128.3 \%$ and $90.0 \%$, respectively). MLG extract at a concentration of $10 \%$ does not have a significant effect on the change in the optical density of the culture. The increase of L, ML and MLG extracts' content in the cultivation medium for up to $30 \%$ is accompanied by a significant increase in the optical density gain of the culture both in comparison with $\mathrm{PC}_{30}$ (SI are $183 \%, 170.7 \%$ and $100.8 \%$, respectively) and in comparison with $\mathrm{PC}_{10}$ (SI are $134.3 \%, 124.2 \%$ and $66.2 \%$, respectively). Addition to the cultivation medium of $\mathrm{L}, \mathrm{ML}$ and $\mathrm{MLG}$ extracts at a concentration of $50 \%$ results in a significant increase of the optical density gain of the culture both in comparison with $\mathrm{PC}_{50}$ (SI are $714.3 \%$, $706.8 \%$ and $394.3 \%$, respectively), and in comparison with $\mathrm{PC}_{10}$ (StI are $112.8 \%, 110.9 \%$ and $29.2 \%$, respectively).

Thus, L, LM and MLG extracts have a significant stimulatory effect on the proliferative activity of B. bifidum. It is noteworthy that at the same concentration in the cultivation medium $\mathrm{L}$ and $\mathrm{LM}$ extracts have more pronounced stimulatory effect on the optical density gain of the B. bifidum culture than MLG extract.

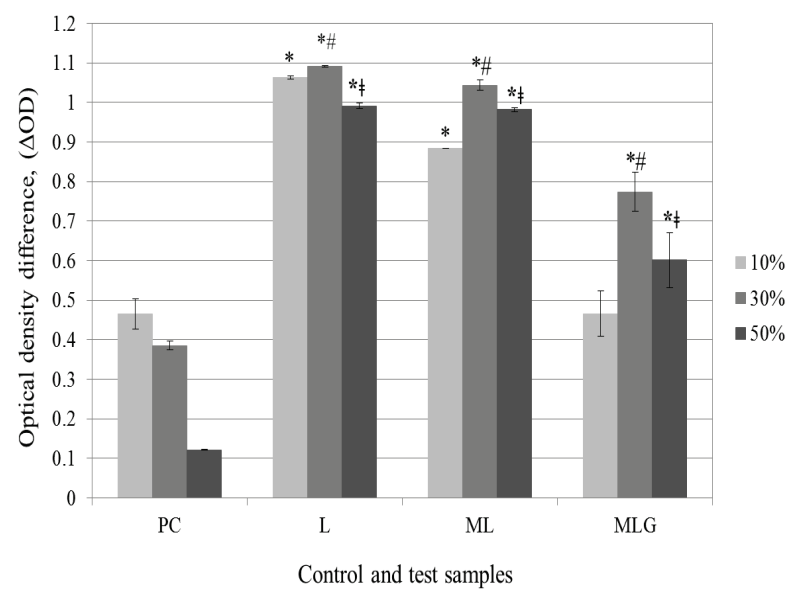

Fig. 1. The effect of cell-free extracts derived from $L$. reuteri on the proliferation of B. bifidum ( $\mathrm{x} \pm \mathrm{SD}$ at $578 \mathrm{~nm}, \mathrm{n}=3$ ): $\mathrm{PC}$ - positive control $\left(\mathrm{PC}_{10} ; \mathrm{PC}_{30} ; \mathrm{PC}_{50}\right) ; \mathrm{L}$ - filtrate of $L$. reuteri disintegrate; $\mathrm{ML}$ - filtrate of $L$. reuteri culture, grown in $L$. reuteri disintegrate; MLG - filtrate of $L$. reuteri culture, grown in L. reuteri disintegrate supplemented with glycerol and glucose; $*$ - the differences are significant compared to the $\mathrm{PC}_{10} ;{ }^{\#}$ - the differences are significant compared to the $\mathrm{PC}_{30} ; \neq-$ the differences are significant compared to the $\mathrm{PC}_{50}, \mathrm{P}<0.05$

As shown in Figure 2, B and MB cell-free extracts in the composition of the cultivation medium at a concentration of $10 \%$ cause a significant increase in the optical density gain of the culture compared to $\mathrm{PC}_{10}$ (SI are $89.8 \%$ and $32.6 \%$, respectively). An increase of $\mathrm{B}$ and $\mathrm{MB}$ extracts' concentration in the cultivation medium of up to $30 \%$ leads to even more significant increase in the optical density gain of the culture compared to $\mathrm{PC}_{30}$ (StI are $135.4 \%$ and $107.7 \%$, respectively) and compared to $\mathrm{PC}_{10}$ (SI are $95 \%$ and $72 \%$, respectively). If cell-free $\mathrm{B}$ and $\mathrm{MB}$ extracts make up $50 \%$ of the cultivation medium, then a significant increase in the optical density gain of the culture is observed both in comparison with $\mathrm{PC}_{50}$ (SI are $613.8 \%$ and $403.5 \%$, respectively) and in comparison with $\mathrm{PC}_{10}$ (SI are $86.6 \%$ and $31.6 \%$, respectively).

The obtained data allow us to conclude that $\mathrm{B}$ and $\mathrm{MB}$ extracts have a significant stimulatory effect on the $B$. bifidum proliferation. It should be noted that with the same concentration in the culture medium B extract has more pronounced stimulatory effect on the proliferation of the B. bifidum culture than $\mathrm{MB}$ extract.

Optical density of the eluate enables us to indirectly judge the biofilm formation by bacteria. An increase in the volume of the $\mathrm{PhS}$ replacing the nutrient medium in the control sample from $10 \%$ to $30 \%$ and from $10 \%$ to $50 \%$ is accompanied by a decrease in the optical density of the eluate by an average of $27 \%$ and $62.9 \%$, respectively (Fig. 3 ). The biofilm formation by culture B. bifidum at $10 \%$ and $30 \%$ content of the $\mathrm{PhS}$ in the cultivation medium is defined as moderate. With an increase in the $\mathrm{PhS}$ content in the cultivation medium up to $50 \%$ the biofilm formation by $B$. bifidum is defined as weak. The addition to the cultiva- tion medium of extract $\mathrm{L}$ at a concentration of $10 \%$ does not signifycantly affect the biofilm formation by bifidobacteria. The inclusion of this cell-free extract in the composition of the cultivation medium at a concentration of $30 \%$ is accompanied by a decrease in the optical density of the eluate compared with $\mathrm{PC}_{30}$ (II is $47.3 \%$, the biofilm formation by B. bifidum is defined as weak). Increasing the concentration of extract $\mathrm{L}$ to $50 \%$ led to a decrease in the optical density of the eluate compared to $\mathrm{PC}_{50}$ (II is $21.4 \%$ ). Optical density of such eluate did not significantly differ from the optical density of the NC (data not shown). ML cell-free extract, when added to cultivation medium at the $10 \%$ concentration, caused a significant increase in the optical density of the eluate compared to $\mathrm{PC}_{10}$ (SI is $28 \%$, the biofilm formation by B. bifidum is defined as strong). $30 \%$ content of the ML extract leads to increase in the optical density of the eluate compared with the $\mathrm{PC}_{30}$ (SI is $49.3 \%$ ). However, there is a tendency to decrease in optical density compared with eluate of samples containing this extract at a concentration of $10 \%$. The biofilm formation by B. bifidum culture at $30 \%$ content of the ML extract in the cultivation medium is defined as moderate. Optical density of the sample eluate, which contains $50 \%$ of the ML extract, is not significantly different from $\mathrm{PC}_{50}$ (biofilm formation by the $B$. bifidum culture is also defined as weak). Adding MLG extract to the culture medium at concentrations of $10 \%, 30 \%$ and $50 \%$ results in the most significant increase in the optical density of the eluates compared with the corresponding control values $\left(\mathrm{PC}_{10}, \mathrm{PC}_{30}, \mathrm{PC}_{50}\right)$. SI are $39.6 \%, 142.6 \%$ and $73.7 \%$, respectively. When the content in the cultivation medium of the MLG extract is $10 \%$ and $30 \%$, the culture goes into the discharge with strong biofilm formation.

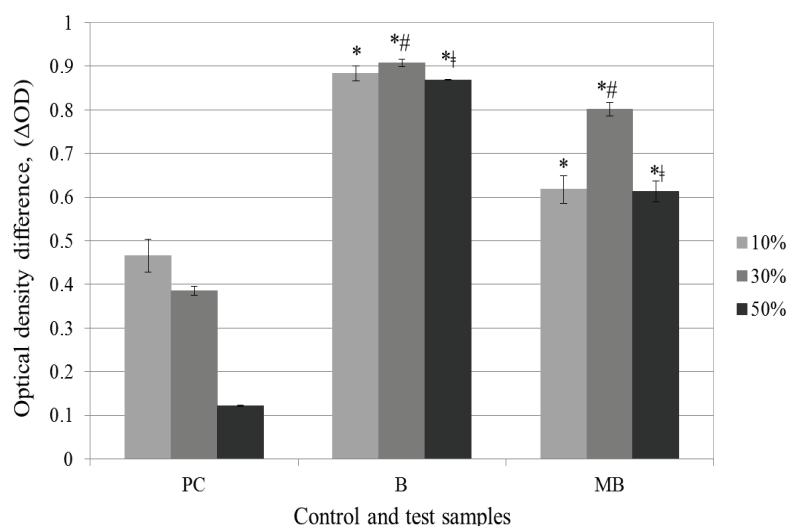

Fig. 2. The effect of cell-free extracts derived from B. bifidum on the proliferation of B. bifidum $(\mathrm{x} \pm \mathrm{SD}$ at $578 \mathrm{~nm}, \mathrm{n}=3)$ : $\mathrm{PC}$ - positive control $\left(\mathrm{PC}_{10}, \mathrm{PC}_{30}, \mathrm{PC}_{50}\right)$; $\mathrm{B}$ - filtrate of $B$. bifidum disintegrate; $\mathrm{MB}$ - filtrate of $B$. bifidum culture, grown in $B$. bifidum disintegrate; * - the differences are significant compared to the $\mathrm{PC}_{10}$;

$\#$ - the differences are significant compared to the $\mathrm{PC}_{30} ; \neq-$ the

differences are significant compared to the $\mathrm{PC}_{50}, \mathrm{P}<0.05$

From the obtained results it follows that the $\mathrm{L}$ extract has an inhibitory effect, while LM and MLG extracts have a stimulatory effect on the biofilm formation by B. bifidum. MLG extract has a more pronounced stimulatory effect on the biofilm formation by B. bifidum than ML extract.

The data presented in Figure 4 suggest that the addition of B and MB cell-free extracts to the cultivation medium at a concentration of $10 \%$ leads to a significant increase in the optical density of the eluates compared to $\mathrm{PC}_{10}$ (SI are $29.8 \%$ and $31.5 \%$ ). An increase in the content of $\mathrm{B}$ and $\mathrm{MB}$ extracts in the cultivation medium up to $30 \%$ is accompanied by the increase in the optical density of the eluates compared to $\mathrm{PC}_{30}$ (StI are 53.0\% and 55.8\%) and at the same time a significant (B) or insignificant (MB) decrease in the optical density of the eluates compared to samples containing the studied extracts at a concentration $10 \%$. Optical density of the eluates of samples containing B and MB extracts at a concentration of $50 \%$ is significantly higher compared to $\mathrm{PC}_{50}$ (SI are $96.3 \%$ and $119.7 \%$ ), but much lower compared to samples containing the studied extracts at a concentration $10 \%$ and $30 \%$.

The biofilm formation by B. bifidum culture at $10 \%$ and $30 \%$ content of $\mathrm{B}$ and $\mathrm{MB}$ extracts in the cultivation medium is defined as strong. 
At $50 \%$ content of $\mathrm{B}$ and $\mathrm{MB}$ extracts in the cultivation medium the biofilm formation by B. bifidum culture is defined as moderate. Thus, B and $\mathrm{MB}$ extracts stimulate biofilm formation by $B$. bifidum. It should be noted that there was no significant difference in the intensity of the stimulatory effect of $\mathrm{B}$ and $\mathrm{MB}$ extracts on the biofilm formation.

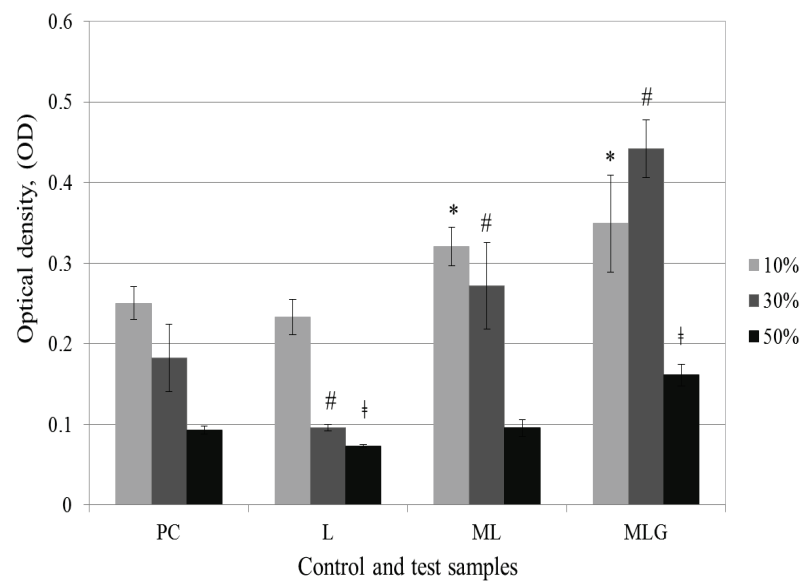

Fig. 3. The effect of cell-free extracts derived from $L$. reuteri on the biofilm formation by B. bifidum ( $\mathrm{x} \pm \mathrm{SD}$ at $630 \mathrm{~nm}, \mathrm{n}=3)$ : see Fig. 1

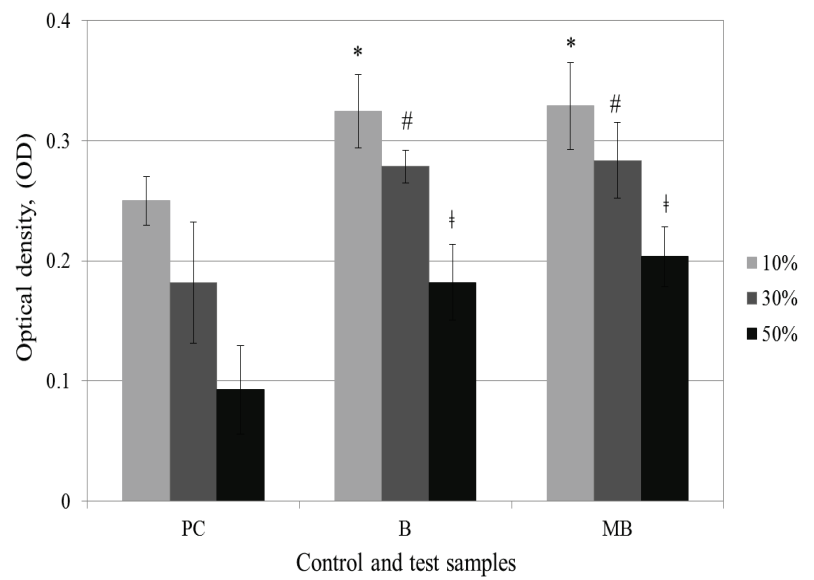

Fig. 4. The effect of cell-free extracts derived from $B$. bifidum on the biofilm formation by $B$. bifidum ( $\mathrm{x} \pm \mathrm{SD}$ at $630 \mathrm{~nm}, \mathrm{n}=3$ ): see Fig. 2

\section{Discussion}

Summarizing the obtained data, it should be noted that all the studied cell-free extracts stimulate the proliferative activity of B. bifidum. According to the degree of stimulatory effect on the $B$. bifidum proliferation, cell-free extracts arranged in ascending order: $\mathrm{MLG}<\mathrm{MB}<\mathrm{B}<$ $\mathrm{ML}<\mathrm{L}$. The stimulation abilities of cell-free extracts are manifested in a concentration dependent way. With the same content in the culture medium, filtrates of disintegrates have a more pronounced stimulatory effect than filtrates of the cultures grown in their own disintegrates. Cellfree extracts from $L$. reuteri ( $\mathrm{L}$ and $\mathrm{ML}$ ) have a more pronounced stimulatory effect than cell-free extracts from $B$. bifidum. The least pronounced stimulatory effect on $B$. bifidum proliferation is observed under the influence of MLG extract. It is known that $L$. reuteri can convert glycerol into the antimicrobial compound reuterin (Spinler et al., 2017). Perhaps the least stimulating effect of the MLG extract is related to the sensitivity of the test culture to reuterin.

Similar results indicating the bifidogenic effect of probiotic derivatives were obtained by other authors. They show that some strains of propionic acid bacteria have ability to produce metabolites, which stimulate the growth of Bifidobacterium strains (Warminska-Radyko et al., 2002). "Bifidogenic effects are widely known, yet the underlying mechanism is poorly understood" (Sarkar \& Mandal, 2016). Currently, we can only assume which components of the complex extracts play the role of a bifidogenic factor.
Possibly, the explanation of the mechanism of action of the obtained extracts should be sought in the method of obtaining the extracts, which determines their composition. To obtain disintegrates, a slow disintegration method was used, in which bacterial cells were subjected to powerful stress and damaging effects, receiving sublethal and lethal damage. During the freeze-thaw stages microorganisms are exposed to thermal, osmotic, thermomechanical, dehydration and rehydration shock. Some cells die. Structural components and metabolites leave the destroyed cells and enter the environment. In the remaining living cells, metabolism is altered. It is known that in response to cold shock, a cascade of cellular reactions, accompanied by a high level of temporary expression of cold-induced proteins (CIPs) occurs. CIPs perform pleiotropic functions, such as the regulation of transcription, translation, and splicing. They have the ability to orchestrate multiple cellular processes, including proliferation and differentiation (Lindquist \& Mertens, 2018). Thus, disintegrate obtained by repeated freezing-thawing of a bacterial suspension contains the structural components of bacterial cells (MAMPs, microbe-associated molecular patterns), bacterial cold shock proteins (DAMPs, damage-associated molecular patterns), which possess powerful bioregulatory potential. Some substances can be catabolized by bifidobacteria and serve as signaling molecules involved in the regulation of gene expression and in the quorum sensing mechanism implementation.

The results of studies by many authors demonstrate that some exopolysaccharides (EPSs) produced by Lactobacillus or Bifidobacterium are able to exert a bifidogenic effect in vitro (Patten \& Laws, 2015; Ryan et al., 2015). Subsequent in vivo studies confirmed the effectiveness of EPSs as a growth factor for bifidobacteria. For example, kefiran, a branched hydrosoluble glucogalactan, heteropolysaccharide produced by $L$. kefiranofaciens, is able to change intestinal and faecal microbiota of BALB/c mice, by increasing the number of bifidobacteria populations (Hamet et al., 2016). One of the possible mechanisms of the beneficial action of kefiran is its ability to induce the expression of gene related to carbohydrates transport and metabolism of B. bifidum PRL2010 (Serafini et al., 2014). It has been shown that lyophilized EPSs produced by lactobacilli have the ability to cause a bifidogenic effect comparable to the action of prebiotic inulin (Sarikaya et al., 2017).

The biosynthesis of these bioactive molecules is different in different genera (Patel et al., 2010). Probiotic EPSs have different chemical structure, degree of branching, sugar composition, molecular weight and glycosidic linkages of each polymer of EPSs (Hamet et al., 2016). The presence and severity of bifidogenic effect depends on physical and chemical properties of EPSs (Polak-Berecka et al., 2013; Hamet et al., 2016). EPSs with a different type of monosaccharide composition stimulate the growth of bifidobacteria at different rates (Sarikaya et al., 2017). The yield, composition and structure of EPSs produced by the same species of microorganism depend on the growth conditions and the composition of the cultivation medium, specifically on the type of the carbohydrate source in the growth medium (Patel et al., 2010; Polak-Berecka et al., 2013). Various probiotic strains synthesize EPSs mixtures of various structures under the same cultivation conditions. The monosaccharide units of homopolysaccharides produced by some strains of $L$. reuteri were determined: levan, reuteran, dextran, mutan. EPSs-producing bacteria are generally not able to destroy their own EPSs. Unrelated microbes are capable to catabolize polysaccharides of other bacteria (Patel et al., 2010). Several studies have demonstrated the resistance of EPSs to biodegradation under conditions of simulating gastric and intestinal digestion (Patten \& Laws, 2015). Therefore, EPSs with a bifidogenic effect can be considered as an alternative to traditional probiotic preparations, which show a low survival level in the gastrointestinal tract. Besides EPSs, the bifidogenic effect is attributed to such structural components and probiotic metabolites as: lipoteichoic acids, short chain fatty acids and bifidocins (Sarkar \& Mandal, 2016; Valdes et al., 2018). Which biologically active components of the studied cell-free extracts have a stimulating effect on the growth of B. bifidum remains to be studied.

Not all studied cell-free extracts stimulate the biofilm formation by B. bifidum. The effect of cell-free extracts on this process depends on their type and concentration. Extract L has a predominantly inhibitory effect on biofilm formation by $B$. bifidum. The most pronounced stimu- 
latory effect on biofilm formation by $B$. bifidum is from an extract MLG. ML, B and MB extracts stimulate this process approximately equally. Currently, it is not possible to accurately determine the mechanism of this stimulating influence. However, we can make theoretical assumptions about the possible mechanisms of the effect of cell-free extracts on this complex biological process. It is thought that EPSs play a key role in bacterial biofilms: they are a major constituent in these matrices, stabilize the 3-D structure of biofilms and minimize intercellular repulsions between bacteria by shielding the electrostatic charges on their surfaces. It is known that excessive EPSs production by one strain of bacteria can significantly enhance the stability of biofilms of other, nonEPS-producing bacteria (Patten \& Laws, 2015). But from these positions it is difficult to explain the mechanism of the inhibitory effect of L extract on biofilm formation by $B$. bifidum, especially taking into account its most pronounced stimulatory effect on the proliferation of a probiotic test culture. The obtained results indicate the existence of a mechanism for inhibiting the biofilm formation that is not associated with inhibition of proliferation.

According to recent studies, bacterial exposure to subinhibitory antimicrobials of many chemically different classes increases biofilm formation. This phenomenon can be explained by relying on the fundamental biomedical paradigm of hormesis, according to which small doses stimulate, and large inhibit biological parameters. This hormetic response can be considered as a quick and nonspecific way to protect the population from chemical threats (Ranieri et al., 2018). We assume that the most pronounced stimulatory effect of MLG extract on biofilm formation by B. bifidum is related precisely to the content in the extract of reuterin, which has antimicrobial activity.

Thus, the mechanisms of the stimulatory effect on proliferation and of the different effects on the B. bifidum biofilm formation of cell-free extracts containing probiotic derivatives are not entirely clear and need to be thoroughly investigated.

\section{Conclusion}

The present study shows that cell-free extracts have the ability to stimulate the growth and differently affect biofilm formation by B. bifidum. The detection of significant bifidogenic effect of the studied cell-free extracts may contribute to their pharmaceutical applications. Cell-free extracts can be used as metabiotics or prebiotics for increasing the survival of the injected probiotic, facilitating its inoculation in the gastrointestinal tract when used together. This study also encourages further careful study of the biochemical composition of cell-free extracts and efforts to clarify the mechanism of their action.

\section{References}

Cicvarek, J., Čurda, L., Elich, O., Dvořáková, E., \& Dvořák, M. (2010). Effect of caseinomacropeptide concentrate addition on the growth of bifidobacteria. Czech Journal of Food Sciences, 28(6), 485-494.

Feng, Q., Chen, W. D., \& Wang, Y. D. (2018). Gut microbiota: An integral moderator in health and disease. Frontiers in Microbiology, 9, 151.

Gagliardi, A., Totino, V., Cacciotti, F., Iebba, V., Neroni, B., Bonfiglio, G., Trancassini, M., Passariello, C., Pantanella, F, \& Schippa, S. (2018). Rebuilding the gut microbiota ecosystem. International Journal of Environmental Research and Public Health, 15(8), 1679 .

Hamet, M. F., Medrano, M., Perez, P. F., \& Abraham, A. G. (2016). Oral administration of kefiran exerts a bifidogenic effect on BALB/c mice intestinal microbiota. Beneficial Microbes, 7(2), 237-246.

In Lee, S. H., Barancelli, G. V., de Camargo, T. M., Corassin, C. H., Rosim, R. E., da Cruz, A. G., Cappato, L. P., \& de Oliveira, C. A. (2017). Biofilm-producing ability of Listeria monocytogenes isolates from Brazilian cheese processing plants. Food Research International, 91, 88-91.

Keith, J. W., \& Pamer, E. G. (2018). Enlisting commensal microbes to resist antibiotic-resistant pathogens. Journal of Experimental Medicine, 2018, jem20180399.

Kho, Z. Y., \& Lal, S. K. (2018). The human gut microbiome-A potential controller of wellness and disease. Frontiers in Microbiology, 9, 1835

Knysh, O. V., Isajenko, O. J., Babych, J. M., Poljans'ka, V. P., Zachepylo, S. V., Kompanijec', A. M., \& Gorbach, T. V. (2018). Sposib oderzhannja biologichno aktyvnyh deryvativ bakterij probiotychnyh shtamiv [Method for obtai- ning biologically active derivatives of bacteria of probiotic strains]. Patent of Ukraine for useful model No 122859. Derzhavne Patentne Vidomstvo Ukrainy, Kyiv (in Ukrainian).

Ku, S., Park, M., Ji, G., \& You, H. (2016). Review on Bifidobacterium bifidum bgn4: Functionality and nutraceutical applications as a probiotic microorganism. International Journal of Molecular Sciences, 17(9), 1544.

Landman, C., \& Quevrain, E. (2016). Gut microbiota: Description, role and pathophysiologic implications. La Revue de MedecineInterne, 37(6), 418-423.

Lindquist, J. A., \& Mertens, P. R. (2018). Cold shock proteins: From cellular mechanisms to pathophysiology and disease. Cell Communication and Signaling, $16(1), 63$.

Litvak, Y., Byndloss, M. X., Tsolis, R. M., \& Bäumler, A. J. (2017). Dysbiotic Proteobacteria expansion: A microbial signature of epithelial dysfunction. Current Opinion in Microbiology, 39, 1-6.

O'Callaghan, A., \& van Sinderen, D. (2016). Bifidobacteria and their role as members of the human gut microbiota. Frontiers in Microbiology, 7, 925.

O'Neill, I., Schofield, Z., \& Hall, L. J. (2017). Exploring the role of the microbiota member Bifidobacterium in modulating immune-linked diseases. Emerging Topics in Life Sciences, 1(4), 333-349.

Patel, A. K., Michaud, P., Singhania, R. R., Soccol, C. R., \& Pandey, A. (2010). Polysaccharides from probiotics: New developments as food additives. Food Technology and Biotechnology, 48(4), 451-463.

Patten, D. A., \& Laws, A. P. (2015). Lactobacillus-produced exopolysaccharides and their potential health benefits: A review. Beneficial Microbes, 6(4), 457-471.

Polak-Berecka, M., Wasko, A., Szwajgier, D., \& Choma, A. (2013). Bifidogenic and antioxidant activity of exopolysaccharides produced by Lactobacillus rhamnosus E/N cultivated on different carbon sources. Polish Journal of Microbiology, 62(2), 81-189.

Quigley, E. M. M. (2016). Bifidobacterium bifidum. In: Floch, M. H., Ringel, Y., \& Walker, W. A. (Eds.). The microbiota in gastrointestinal pathophysiology: Implications for human health, prebiotics, probiotics, and dysbiosis. Elsevier Inc. Part B, Chaptter 14. Pp. 131-133.

Ranieri, M. R., Whitchurch, C. B., \& Burrows, L. L. (2018). Mechanisms of biofilm stimulation by subinhibitory concentrations of antimicrobials. Current Opinion in Microbiology, 45, 164-169.

Richards, J. L., Yap, Y. A., McLeod, K. H., Mackay, C. R., \& Mariño, E. (2016). Dietary metabolites and the gut microbiota: An alternative approach to control inflammatory and autoimmune diseases. Clinical and Translational Immunology, 5(5), e82.

Rivière, A., Selak, M., Lantin, D., Leroy, F., \& de Vuyst, L. (2016). Bifidobacteria and butyrate-producing colon bacteria: Importance and strategies for their stimulation in the human gut. Frontiers in Microbiology, 28(7), 979.

Ryan, P. M., Ross, R. P., Fitzgerald, G. F., Caplice, N. M., \& Stanton, C. (2015). Sugar-coated: Exopolysaccharide producing lactic acid bacteria for food and human health applications. Food and Function, 6(3), 679-693.

Sarikaya, H., Aslim, B., \& Yuksekdag, Z. (2017). Assessment of anti-biofilm activity and bifidogenic growth stimulator (BGS) effect of lyophilized exopolysaccharides (1-EPSs) from Lactobacilli strains. International Journal of Food Properties, 20(2), 362-371.

Sarkar, A., \& Mandal, S. (2016). Bifidobacteria - insight into clinical outcomes and mechanisms of its probiotic action. Microbiological Research, 192, 159-171.

Serafini, F., Turroni, F., Ruas-Madiedo, P., Lugli, G. A., Milani, C., Duranti, S., Zamboni, N., Bottacini, F., Sinderen, D., Margolles, A., \& Ventura, M. (2014). Kefir fermented milk and kefiran promote growth of Bifidobacterium bifidum PRL2010 and modulate its gene expression. International Journal of Food Microbiology, 178, 50-59.

Shenderov, B. A. (2013). Metabiotics: Novel idea or natural development of probiotic conception. Microbial Ecology in Health and Disease, 24(1), 20399.

Singh, A., Vishwakarma, V., \& Singhal, B. (2018). Metabiotics: The functional metabolic signatures of probiotics: Current state-of-art and future research priorities. Advances in Bioscience and Biotechnology, 9, 147-189.

Spinler, J., Auchtung, J., Brown, A., Boonma, P., Oezguen, N., \& Ross, C., Luna, R. A., Runge, J., Versalovic, J., Peniche, A., Dann, S. M., Britton, R. A., Haag, A., \& Savidge, T. C. (2017). Next-generation probiotics targeting Clostridium difficile through precursor-directed antimicrobial biosynthesis. Infection and Immunity, 85(10), e00303-17.

Turroni, S., Brigidi, P., Cavalli, A., \& Candela, M. (2017). Microbiota - host transgenomic metabolism, bioactive molecules from the inside: Miniperspective. Journal of Medicinal Chemistry, 61(1), 47-61.

Valdes, A. M., Walter, J., Segal, E., \& Spector, T. D. (2018). Role of the gut microbiota in nutrition and health. British Medical Journal, 361, k2179.

Wang, B., Yao, M., Lv, L., Ling, Z., \& Li, L. (2017). The human microbiota in health and disease. Engineering, 3(1), 71-82.

Warminska-Radyko, I., Laniewska-Moroz, L., \& Babuchowski, A. (2002). Possibilities for stimulation of Bifidobacterium growth by propionibacteria. Le Lait, 82(1), 113-121. 